\title{
Normal Families concerning Polynomials and Shared Values
}

\author{
Xin-Li Wang ${ }^{1}$ and Ning Cui ${ }^{2}$ \\ ${ }^{1}$ College of Science, University of Shanghai for Science and Technology, Shanghai 200093, China \\ ${ }^{2}$ School of Mathematical Sciences, South China Normal University, Guangzhou 510631, China \\ Correspondence should be addressed to Xin-Li Wang; xlwang602@163.com
}

Received 8 July 2013; Revised 14 September 2013; Accepted 15 September 2013

Academic Editor: Wen-Xiu Ma

Copyright (C) 2013 X.-L. Wang and N. Cui. This is an open access article distributed under the Creative Commons Attribution License, which permits unrestricted use, distribution, and reproduction in any medium, provided the original work is properly cited.

We study the problem of normal families of meromorphic functions concerning polynomials and shared values. We prove that a family $\mathscr{F}$ of meromorphic functions in a domain D is normal if, for each function $f \in \mathscr{F}, P(f(z)) f^{(k)}(z)=a \Leftrightarrow f^{(k)}(z)=b$, where $P$ is a polynomial with the origin as zero, $k$ is a positive integer, and $a(\neq 0), b$ are two finite constants.

\section{Introduction and Main Results}

Let $\mathrm{D}$ be a domain in $\mathbb{C}$ and let $\mathscr{F}$ be a family of meromorphic functions defined in D. $\mathscr{F}$ is said to be normal in D, in the sense of Montel, if, for any sequence $\left\{f_{n}\right\} \in \mathscr{F}$, there exists a subsequence $\left\{f_{n_{j}}\right\}$ such that $\left\{f_{n_{j}}\right\}$ converges spherically locally uniformly in $\mathrm{D}$ to a meromorphic function or $\infty$ (see $[1])$.

Let $f$ and $g$ be meromorphic functions in $\mathrm{D}$, and let $a$ and $b$ be complex numbers. If $g(z)=b$ whenever $f(z)=a$, we write

$$
f(z)=a \Longrightarrow g(z)=b \text {. }
$$

If $f(z)=a \Rightarrow g(z)=b$ and $g(z)=b \Rightarrow f(z)=a$, we write

$$
f(z)=a \Longleftrightarrow g(z)=b .
$$

If $f(z)=a \Leftrightarrow g(z)=a$, we say that $f$ and $g$ share $a$ on D.

In 1992, Schwick [2] firstly found a connection between normality criteria and shared values as follows.

Theorem A. Let $\mathscr{F}$ be a family of meromorphic functions in $D$, and let $a_{1}, a_{2}, a_{3}$ be distinct complex numbers. If, for each $f \in \mathscr{F}$, $f$ and $f^{\prime}$ share $a_{i}(i=1,2,3)$, then $\mathscr{F}$ is normal in $D$.

In recent years, more results about normality criteria concerning shared values have been found (see [3-5]). The next theorem was proved by Fang and Zalcman in [4], which was an important normality criteria concerning shared values.

Theorem B. Let $\mathscr{F}$ be a family of meromorphic functions in $D$; let $k$ be a positive integer; let $a \neq 0$ and $b$ be two finite values. If, for every $f \in \mathscr{F}$, all zeros of fhave multiplicity at least $k$ and $f(z) f^{(k)}(z)=a \Leftrightarrow f^{(k)}(z)=b$, then $\mathscr{F}$ is normal in $D$.

Based on the idea in [3], we will improve and extend Theorem B in the following results.

Theorem 1. Let $\mathscr{F}$ be a family of meromorphic functions in D; let $k$ be a positive integer; let $a \neq 0$ and $b$ be two finite values. Let $P$ be a polynomial with the origin as zero. If, for every $f \in \mathscr{F}$, all zeros of $f$ have multiplicity at least $k$ and $P(f(z)) f^{(k)}(z)=$ $a \Leftrightarrow f^{(k)}(z)=b$, then $\mathscr{F}$ is normal in $D$.

Example 2. Let $D=\{z:|z|<1\}, P(z)=z^{2}, \mathscr{F}=\left\{f_{n}(z)\right.$ : $\left.f_{n}(z)=\left(1 / n^{2}\right)\left(e^{n z}+e^{-n z}-2\right)\right\}$. Then, all zeros of $f_{n}(z)$ have multiplicity at least 2 . For each $f_{n}(z) \in \mathscr{F}$, we have $f_{n}^{\prime}(z)=$ $(1 / n)\left(e^{n z}-e^{-n z}\right), f_{n}^{\prime \prime}(z)=e^{n z}+e^{-n z}$. Thus, $P\left(f_{n}(z)\right) f_{n}^{\prime \prime}(z)=$ $\left(1 / n^{4}\right)\left(e^{n z}+e^{-n z}-2\right)^{2}\left(e^{n z}+e^{-n z}\right) \cdot P\left(f_{n}(z)\right) f_{n}^{\prime \prime}(z)=0 \Rightarrow$ $f_{n}^{\prime \prime}(z)=2$, but $\mathscr{F}$ is not normal in $\mathrm{D}$. This shows that the condition $a \neq 0$ is necessary.

Theorem 3. Let $\mathscr{F}$ be a family of meromorphic functions in D; let $k$ be a positive integer; let $a \neq 0$ and $b$ be two finite values. Let $P$ be a polynomial with the origin as zero and $\operatorname{deg} P \geq 2$, 
which has at least one multiple zero. If, for every $f \in \mathscr{F}$, all zeros of fhave multiplicity at least $k$ and $P(f(z)) f^{(k)}(z)=a \Rightarrow$ $f^{(k)}(z)=b$, then $\mathscr{F}$ is normal in $D$.

\section{Some Lemmas}

In order to prove our results, we need the following lemmas.

Lemma 1 (see [6]). Let $\mathscr{F}$ be a family of meromorphic functions on the unit disc $\Delta$ satisfying all zeros of functions in $\mathscr{F}$ which have multiplicity $\geq p$ and all poles of functions in $\mathscr{F} \geq q$. Let $\alpha$ be a real number satisfying $-p<\alpha<q$. Then, $\mathscr{F}$ is not normal at a point $z_{0} \in \Delta$ if and only if there exist

(i) points $z_{n} \in \Delta, z_{n} \rightarrow z_{0}$;

(ii) positive numbers $\rho_{n}, \rho_{n} \rightarrow 0$;

(iii) functions $f_{n} \in \mathscr{F}$ such that $g_{n}(\xi)=\rho_{n}^{\alpha} f_{n}\left(z_{n}+\rho_{n} \xi\right) \rightarrow$ $g(\xi)$ spherically uniformly on each compact subset of $\mathbb{C}$, where $g$ is a nonconstant meromorphic function satisfying the zeros of $g$ which are of multiplicities $\geq p$ and the poles of $g$ which are of multiplicities $\geq q$. Moreover, the order of $g$ is not greater than 2.

Here, $g^{\#}=\left|g^{\prime}(\xi)\right| /\left(1+|g(\xi)|^{2}\right)$ is the spherical derivative.

Lemma 2 (see [7]). Let $\mathscr{F}$ be a family of meromorphic functions on the complex plane $\mathbb{C}$. If the spherical derivative $f^{\#}$ of $f$ is bounded on $\mathbb{C}$, then the order of $f$ is at most two. Especially, when $f$ is an entire function on the complex plane $\mathbb{C}$, then the order of $f$ is at most one.

Lemma 3 (see [8]). Let $f$ be a transcendental meromorphic function on the complex plane $\mathbb{C}$, and let $n$ be a positive integer. Then $f^{n} f^{\prime}$ assumes every nonzero finite complex value infinitely often.

Lemma 4 (see [9]). Let $n \geq 2$ and $k$ be two positive integers, and let $f$ be a transcendental meromorphic function. Then $f^{n} f^{(k)}$ assumes every nonzero finite complex value infinitely often.

Lemma 5 (see [5]). Let b be a nonzero finite complex number, let $n, k$ be two positive integers, and let $f(z)$ be a nonconstant rational function; all of whose zeros have multiplicities at least $k$. For the polynomial case, if $n \geq 2$ or $k \geq 2$, then $f^{n} f^{(k)}-b$ has at least two distinct zeros; for the nonpolynomial rational case, the conclusion holds if $n \geq 2$.

\section{Proof of Theorems}

We only prove Theorem 1, and the proof of Theorem 3 is similar to the second case in the proof of Theorem 1.

Next, we consider two cases.

Case 1. Suppose the origin is a simple zero of $P(z)$. We assume that $P(z)=z^{n}+a_{n-1} z^{n-1}+\cdots+a_{1} z$, where $a_{1} \neq 0$. Without loss of generality, we may assume that $\mathrm{D}=\Delta=\{z:|z|<1\}$. Since normality is a local property, it is enough to show that $\mathscr{F}$ is normal at each point of D. Suppose, on the contrary, that $\mathscr{F}$ is not normal at $z_{0} \in \mathrm{D}$. By Lemma 1 (with $\alpha=-k / 2$ ), there exist functions $f_{j} \in \mathscr{F}$, points $z_{j} \rightarrow z_{0}$, and positive numbers $\rho_{j} \rightarrow 0$ such that

$$
g_{j}(\xi)=\rho_{j}^{-k / 2} f_{j}\left(z_{j}+\rho_{j} \xi\right) \longrightarrow g(\xi)
$$

converges spherically uniformly on compact subsets of $\mathbb{C}$, where $g(\xi)$ is a nonconstant meromorphic function on $\mathbb{C}$ and all of whose zeros have multiplicity at least $k$. Moreover, the order of $g(\xi)$ is at most 2. Therefore, we have

$$
\begin{aligned}
P( & \left.f_{j}\left(z_{j}+\rho_{j} \xi\right)\right) f_{j}^{(k)}\left(z_{j}+\rho_{j} \xi\right)-a \\
= & f_{j}^{n}\left(z_{j}+\rho_{j} \xi\right)+a_{n-1} f_{j}^{n-1}\left(z_{j}+\rho_{j} \xi\right) \\
& \left.+\cdots+a_{1} f_{j}\left(z_{j}+\rho_{j} \xi\right)\right] f_{j}^{(k)}\left(z_{j}+\rho_{j} \xi\right)-a \\
=[ & \rho_{j}^{(n-1) k / 2} g_{j}^{n}(\xi)+a_{n-1} \rho_{j}^{(n-2) k / 2} g_{j}^{n-1}(\xi)+\cdots \\
& \left.+a_{1} g_{j}(\xi)\right] g_{j}^{(k)}(\xi)-a \longrightarrow a_{1} g g^{(k)}-a
\end{aligned}
$$

uniformly on compact subsets of $\mathbb{C}$ disjoint from the poles of g.

We claim (i) $a_{1} g g^{(k)} \neq a$; (ii) $g^{(k)} \neq 0$.

(i) Suppose that $a_{1} g\left(\xi_{0}\right) g^{(k)}\left(\xi_{0}\right)=a$, then $g\left(\xi_{0}\right) \neq \infty$. If $a_{1} g g^{(k)} \equiv a, g$ is an entire function and $g \neq 0$. By Lemma 2, the order of $g$ is at most 1. Assume $g(\xi)=e^{c \xi+d}$, where $c \neq 0$ and $d$ are two constants. But $a_{1} g g^{(k)}=a_{1} c^{k} e^{2 c \xi+2 d} \neq \equiv a$, which is a contradiction. Therefore, we have $a_{1} g g^{(k)} \neq \equiv \quad a$. According to Hurwitz's theorem, there exists a sequence $\xi_{j}, \xi_{j} \rightarrow$ $\xi_{0}$ such that $P\left(f_{j}\left(z_{j}+\rho_{j} \xi_{j}\right)\right) f_{j}^{(k)}\left(z_{j}+\rho_{j} \xi_{j}\right)=a$ for $j$ sufficiently large. It follows that $f_{j}^{(k)}\left(z_{j}+\rho_{j} \xi_{j}\right)=b$, so that

$$
g_{j}^{(k)}\left(\xi_{j}\right)=\rho_{j}^{k / 2} f_{j}^{(k)}\left(z_{j}+\rho_{j} \xi_{j}\right)=\rho_{j}^{k / 2} b .
$$

Thus

$$
g^{(k)}\left(\xi_{0}\right)=\lim _{j \rightarrow \infty} g_{j}^{(k)}\left(\xi_{j}\right)=0,
$$

which contradicts $a_{1} g\left(\xi_{0}\right) g^{(k)}\left(\xi_{0}\right)=a \neq 0$. This proves (i).

(ii) Suppose that $g^{(k)}\left(\xi_{0}\right)=0$, then $g\left(\xi_{0}\right) \neq \infty$. If $g^{(k)} \equiv 0$, $g$ is a polynomial whose degree is less than $k-1$, which contradicts that all zeros of $g$ have multiplicity at least $k$. So that $g^{(k)} \neq \equiv$. Since $g_{j}^{(k)}(\xi)-\rho_{j}^{k / 2} b=$ $\rho_{j}^{k / 2}\left(f_{j}^{(k)}\left(z_{j}+\rho_{j} \xi\right)-b\right) \rightarrow g^{(k)}(\xi)$, by Hurwitz's theorem, there exists a sequence $\xi_{j}, \xi_{j} \rightarrow \xi_{0}$ such that $f_{j}^{(k)}\left(z_{j}+\rho_{j} \xi_{j}\right)=b$ for $j$ sufficiently large. It follows that $P\left(f_{j}\left(z_{j}+\rho_{j} \xi_{j}\right)\right) f_{j}^{(k)}\left(z_{j}+\rho_{j} \xi_{j}\right)=a$, so that

$$
\begin{gathered}
\lim _{j \rightarrow \infty} P\left(f_{j}\left(z_{j}+\rho_{j} \xi_{j}\right)\right) f_{j}^{(k)}\left(z_{j}+\rho_{j} \xi_{j}\right) \\
=a_{1} g\left(\xi_{0}\right) g^{(k)}\left(\xi_{0}\right)=a \neq 0,
\end{gathered}
$$

which contradicts $g^{(k)}\left(\xi_{0}\right)=0$. This proves (ii). 
Next, we assume that $g$ is a transcendental meromorphic function. When $k=1$, by Lemma $3, g g^{(k)}-\left(a / a_{1}\right)$ assumes every nonzero finite complex value infinitely often, a contradiction. When $k \geq 2$, by Nevanlinna's second fundamental theorem,

$$
\begin{aligned}
T & \left(r, g g^{(k)}\right) \\
& \leq \bar{N}(r, g)+\bar{N}\left(r, \frac{1}{g g^{(k)}}\right)+\bar{N}\left(r, \frac{1}{g g^{(k)}-\left(a / a_{1}\right)}\right) \\
& \leq \bar{N}(r, g)+\bar{N}\left(r, \frac{1}{g}\right)+S(r, g),
\end{aligned}
$$

where $S(r, g)=o(T(r, g))$.

By Nevanlinna's first fundamental theorem, we have

$$
\begin{aligned}
T\left(r, g g^{(k)}\right) & \geq \frac{1}{2}\left[N\left(r, g g^{(k)}\right)+N\left(r, \frac{1}{g g^{(k)}}\right)\right]+O(1) \\
& \geq \frac{k+2}{2} \bar{N}(r, g)+\bar{N}\left(r, \frac{1}{g}\right)+S(r, g) .
\end{aligned}
$$

Combining (8) with (9), we have

$$
\bar{N}(r, g)=S(r, g) .
$$

According to logarithmic derivative theorem,

$$
\begin{aligned}
m\left(r, \frac{1}{g^{2}}\right) & =m\left(r, \frac{g^{(k)}}{g} \frac{1}{g g^{(k)}}\right) \\
& \leq m\left(r, \frac{g^{(k)}}{g}\right)+m\left(r, \frac{1}{g g^{(k)}}\right) \\
& \leq m\left(r, \frac{1}{g g^{(k)}}\right)+S(r, g),
\end{aligned}
$$

we have

$$
\begin{aligned}
& m\left(r, \frac{1}{g^{2}}\right)+m\left(r, \frac{1}{g g^{(k)}-\left(a / a_{1}\right)}\right) \\
& \leq m\left(r, \frac{1}{g g^{(k)}}\right)+m\left(r, \frac{1}{g g^{(k)}-\left(a / a_{1}\right)}\right)+S(r, g) \\
& \leq m\left(r, \frac{1}{g g^{(k)}+\left(1 /\left(g g^{(k)}-\left(a / a_{1}\right)\right)\right)}\right)+S(r, g)
\end{aligned}
$$

$$
\begin{aligned}
\leq & m\left(r,\left[\left(\frac{\left(g g^{(k)}\right)^{\prime}}{g g^{(k)}}+\frac{\left(g g^{(k)}\right)^{\prime}}{g g^{(k)}-\left(a / a_{1}\right)}\right) \frac{1}{\left(g g^{(k)}\right)^{\prime}}\right]\right) \\
& +S(r, g) \\
\leq & m\left(r, \frac{1}{\left(g g^{(k)}\right)^{\prime}}\right)+S(r, g) \\
\leq & T\left(r,\left(g g^{(k)}\right)^{\prime}\right)-N\left(r, \frac{1}{\left(g g^{(k)}\right)^{\prime}}\right)+S(r, g) \\
\leq & \bar{N}(r, g)+T\left(r,\left(g g^{(k)}\right)^{\prime}\right)-N\left(r, \frac{1}{\left(g g^{(k)}\right)^{\prime}}\right) \\
& +S(r, g) .
\end{aligned}
$$

It follows that

$$
\begin{aligned}
& 2 T(r, g) \\
& \leq \bar{N}(r, g)+N\left(r, \frac{1}{g^{2}}\right)+N\left(r, \frac{1}{g g^{(k)}-\left(a / a_{1}\right)}\right) \\
&-N\left(r, \frac{1}{\left(g g^{(k)}\right)^{\prime}}\right)+S(r, g) \\
& \leq \bar{N}(r, g)+(k+1) \bar{N}\left(r, \frac{1}{g}\right) \\
&+\bar{N}\left(r, \frac{1}{g g^{(k)}-\left(a / a_{1}\right)}\right)+S(r, g) .
\end{aligned}
$$

Since $g g^{(k)} \neq a / a_{1}$ and the zeros of $g$ have multiplicity exactly $k(\geq 2)$, we have by (10) and (13)

$$
\begin{aligned}
2 T(r, g) & \leq \frac{k+1}{k} N\left(r, \frac{1}{g}\right)+S(r, g) \\
& \leq \frac{3}{2} N\left(r, \frac{1}{g}\right)+S(r, g) \\
& \leq \frac{3}{2} T(r, g)+S(r, g) .
\end{aligned}
$$

Thus $T(r, g)=S(r, g)$, a contradiction. Hence $g$ is a rational function.

Now, suppose that $g$ is a nonpolynomial rational function. Let

$$
g(\xi)=a_{n} \xi^{n}+a_{n-1} \xi^{n-1}+\cdots+a_{1} \xi+a_{0}+\frac{q(\xi)}{p(\xi)},
$$

where $a_{0}, a_{1}, \ldots, a_{n}$ are constants such that $a_{n} \neq 0$ and $p$ and $q\left(\begin{array}{l}\neq \\ 0\end{array}\right)$ are coprime polynomials with $\operatorname{deg} q<\operatorname{deg} p$ and $\operatorname{deg} p \geq 1$. If $n \geq k$,

$$
\begin{aligned}
g^{(k)}(\xi)= & a_{n} n(n-1) \cdots(n-k+1) \xi^{n-k} \\
& +\cdots+a_{k} k !+\frac{q_{k}(\xi)}{p_{k}(\xi)},
\end{aligned}
$$


where $p_{k}(\xi)$ and $q_{k}(\xi)$ are coprime polynomials and, by induction, $\operatorname{deg} q_{k}<\operatorname{deg} p_{k}$. Then, by (16), $g^{(k)}(\xi)=0$ has solutions, a contradiction. Hence $n<k$. Thus, we have

$$
\begin{gathered}
g(\xi)=a_{l} \xi^{l}+a_{l-1} \xi^{l-1}+\cdots+a_{1} \xi+a_{0}+\frac{q(\xi)}{p(\xi)} \\
\left(l \leq k-1, a_{l} \neq 0\right), \\
g^{(k)}(\xi)=\frac{q_{k}(\xi)}{p_{k}(\xi)} .
\end{gathered}
$$

Since $g^{(k)} \neq 0, q_{k}(\xi)$ is a nonzero constant $A$. Since $\operatorname{deg} P \geq 1$, $g$ must have a pole in the finite plane. It follows that $g^{(k)}$ has a finite pole of order at least $k+1$; thus, $\operatorname{deg} p_{k} \geq k+1$,

$$
\begin{aligned}
& a_{1} g(\xi) g^{(k)}(\xi) \\
& \quad=a_{1}\left(a_{l} \xi^{l}+a_{l-1} \xi^{l-1}+\cdots+a_{1} \xi+a_{0}+\frac{q(\xi)}{p(\xi)}\right) \frac{A}{p_{k}(\xi)} \\
& =A a_{1} \frac{\left(a_{l} \xi^{l}+a_{l-1} \xi^{l-1}+\cdots+a_{1} \xi+a_{0}\right) p(\xi)+q(\xi)}{p(\xi) p_{k}(\xi)} .
\end{aligned}
$$

Then, the equation $a_{1} g(\xi) g^{(k)}(\xi)=a$ has solutions, which contradict $a_{1} g(\xi) g^{(k)}(\xi) \neq a$.

Finally, suppose that $g$ is a polynomial. Since $g^{(k)} \neq 0$ and the zeros of $g$ are of multiplicity $k$, we have $g^{(k)}(\xi)=c_{1}(\xi-$ $\left.c_{2}\right)^{k}$, where $c_{1} \neq 0$. Thus, $a_{1} g(\xi) g^{(k)}(\xi)-a=0$ has solutions, a contradiction.

Case 2. Suppose the origin is a multiple zero of $P(z)$. Without loss of generality, we assume that $\mathrm{D}=\Delta=\{z:|z|<1\}$. Let $P(z)=z^{n}+a_{n-1} z^{n-1}+\cdots+a_{q} z^{q}$, where $q \geq 2$ is a positive integer and $a_{n-1}, \ldots, a_{q}(\neq 0)$ are constants. Suppose $\mathscr{F}$ is not normal at $z_{0} \in \mathrm{D}$. By Lemma 1 (with $\alpha=-k /(q+1)$ ), there exist functions $f_{j} \in \mathscr{F}$, points $z_{j} \rightarrow z_{0}$, and positive numbers $\rho_{j} \rightarrow 0$ such that

$$
g_{j}(\xi)=\rho_{j}^{-k /(q+1)} f_{j}\left(z_{j}+\rho_{j} \xi\right) \longrightarrow g(\xi)
$$

converges spherically uniformly on compact subsets of $\mathbb{C}$, where $g(\xi)$ is a nonconstant meromorphic function on $\mathbb{C}$, and all of whose zeros have multiplicity at least $k$. Moreover, the order of $g(\xi)$ is at most 2 . We have

$$
\begin{aligned}
P\left(f_{j}\left(z_{j}+\rho_{j} \xi\right)\right) f_{j}^{(k)}\left(z_{j}+\rho_{j} \xi\right)-a \\
=\left[f_{j}^{n}\left(z_{j}+\rho_{j} \xi\right)+a_{n-1} f_{j}^{n-1}\left(z_{j}+\rho_{j} \xi\right)\right. \\
\left.\quad+\cdots+a_{q} f_{j}^{q}\left(z_{j}+\rho_{j} \xi\right)\right] f_{j}^{(k)}\left(z_{j}+\rho_{j} \xi\right)-a \\
=\left[\rho_{j}^{(n-q) k /(q+1)} g_{j}^{n}(\xi)+a_{n-1} \rho_{j}^{(n-q-1) k /(q+1)} g_{j}^{n-1}(\xi)\right. \\
\left.\quad+\cdots+a_{q} g_{j}^{q}(\xi)\right] g_{j}^{(k)}(\xi)-a \longrightarrow a_{q} g^{q} g^{(k)}-a
\end{aligned}
$$

uniformly on compact subsets of $\mathbb{C}$ disjoint from the poles of $g$. If $a_{q} g^{q} g^{(k)} \equiv a, g$ has no zeros and poles. That is to say, $g$ is an entire function and $g \neq 0$. Since the spherical derivative of $g$ is bounded, by Lemma 2 , we have $\operatorname{ord}(g) \leq$ 1. Hence suppose $g(\xi)=e^{c \xi+d}$, where $c(\neq 0)$ and $d$ are constants. Thus, $a_{q} g^{q} g^{(k)}=a_{q} c^{k} e^{(q+1) c \xi+(q+1) d} \quad \neq \equiv \quad a$, a contradiction. By Lemmas 4 and $5, a_{q} g^{q} g^{(k)}-a$ has zeros. Suppose $a_{q} g^{q}\left(\xi_{0}\right) g^{(k)}\left(\xi_{0}\right)=a$. Since $a_{q} g^{q} g^{(k)} \not \equiv a$, by Hurwitz's theorem, there exists a sequence $\xi_{j}, \xi_{j} \rightarrow \xi_{0}$ such that (for $j$ sufficiently large)

$$
\begin{aligned}
P( & \left.f_{j}\left(z_{j}+\rho_{j} \xi_{j}\right)\right) f_{j}^{(k)}\left(z_{j}+\rho_{j} \xi_{j}\right)-a \\
=[ & f_{j}^{n}\left(z_{j}+\rho_{j} \xi_{j}\right)+a_{n-1} f_{j}^{n-1}\left(z_{j}+\rho_{j} \xi_{j}\right) \\
& \left.\quad+\cdots+a_{q} f_{j}^{q}\left(z_{j}+\rho_{j} \xi_{j}\right)\right] f_{j}^{(k)}\left(z_{j}+\rho_{j} \xi_{j}\right)-a=0 .
\end{aligned}
$$

It follows that $f_{j}^{(k)}\left(z_{j}+\rho_{j} \xi_{j}\right)=b$, so that

$$
g_{j}^{(k)}\left(\xi_{j}\right)=\rho_{j}^{q k /(q+1)} f_{j}^{(k)}\left(z_{j}+\rho_{j} \xi_{j}\right)=\rho_{j}^{q k /(q+1)} b .
$$

Thus,

$$
g^{(k)}\left(\xi_{0}\right)=\lim _{j \rightarrow \infty} g_{j}^{(k)}\left(\xi_{j}\right)=0
$$

which contradicts $a_{q} g^{q}\left(\xi_{0}\right) g^{(k)}\left(\xi_{0}\right)=a \neq 0$. This completes the proof.

\section{Acknowledgment}

This paper is supported by the National Natural Science Foundation of China (no. 61074016).

\section{References}

[1] W. K. Hayman, Meromorphic Functions, Clarendon Press, Oxford, UK, 1964.

[2] W. Schwick, "Sharing values and normality," Archiv der Mathematik, vol. 59, no. 1, pp. 50-54, 1992.

[3] C. L. Lei and M. L. Fang, "Normality and shared values concerning differential polynomials," Science China, vol. 53, no. 3, pp. 749-754, 2010.

[4] M. L. Fang and L. Zalcman, "Normal families and shared values of meromorphic functions II," Computational Methods and Function Theory, vol. 1, no. 1, pp. 289-299, 2001.

[5] X. Wu and Y. Xu, "Normal families of meromorphic functions and shared values," Monatshefte für Mathematik, vol. 165, no. 34, pp. 569-578, 2012.

[6] L. Zalcman, "Normal families: new perspectives," Bulletin of the American Mathematical Society, vol. 35, no. 3, pp. 215-230, 1998.

[7] J. Clunie and W. K. Hayman, "The spherical derivative of integral and meromorphic functions," Commentarii Mathematici Helvetici, vol. 40, pp. 117-148, 1966.

[8] W. Bergweiler and A. Eremenko, "On the singularities of the inverse to a meromorphic function of finite order," Revista Matemática Iberoamericana, vol. 11, no. 2, pp. 355-373, 1995.

[9] N. Steinmetz, "Über die nullstellen von differentialpolynomen," Mathematische Zeitschrift, vol. 176, no. 2, pp. 255-264, 1981. 


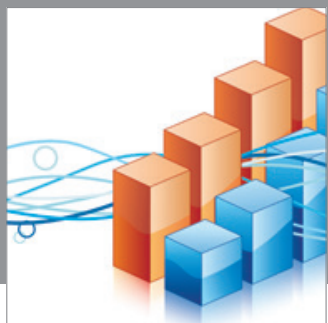

Advances in

Operations Research

mansans

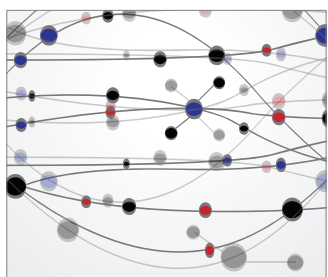

The Scientific World Journal
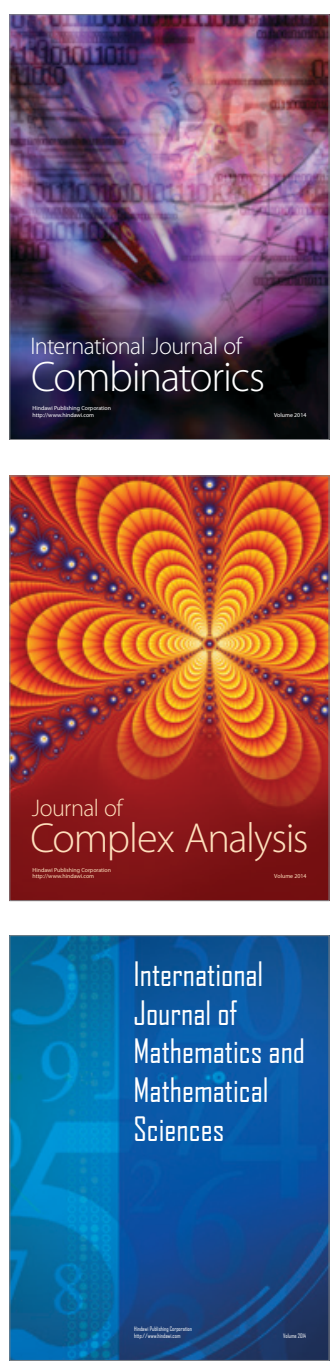
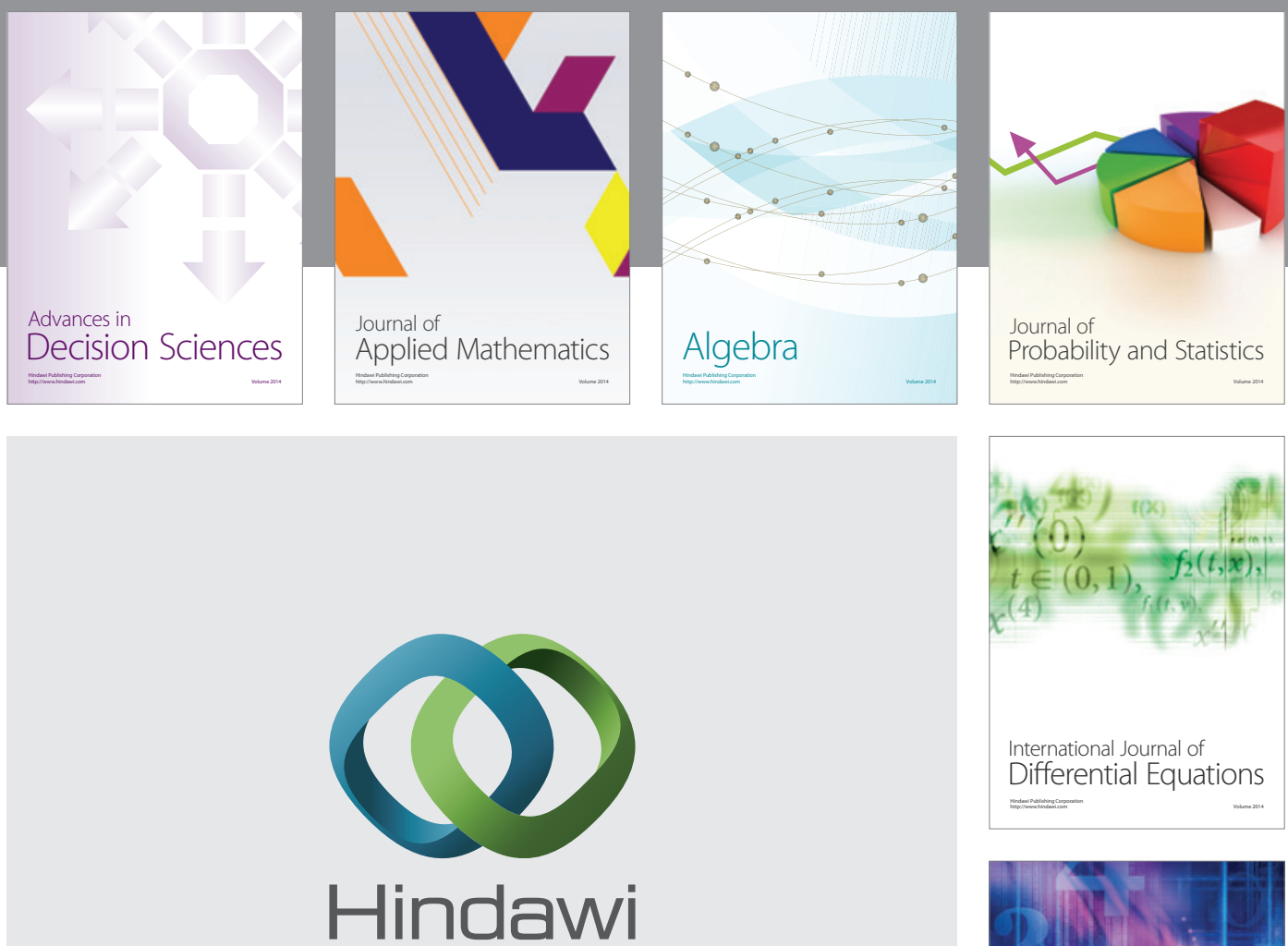

Submit your manuscripts at http://www.hindawi.com
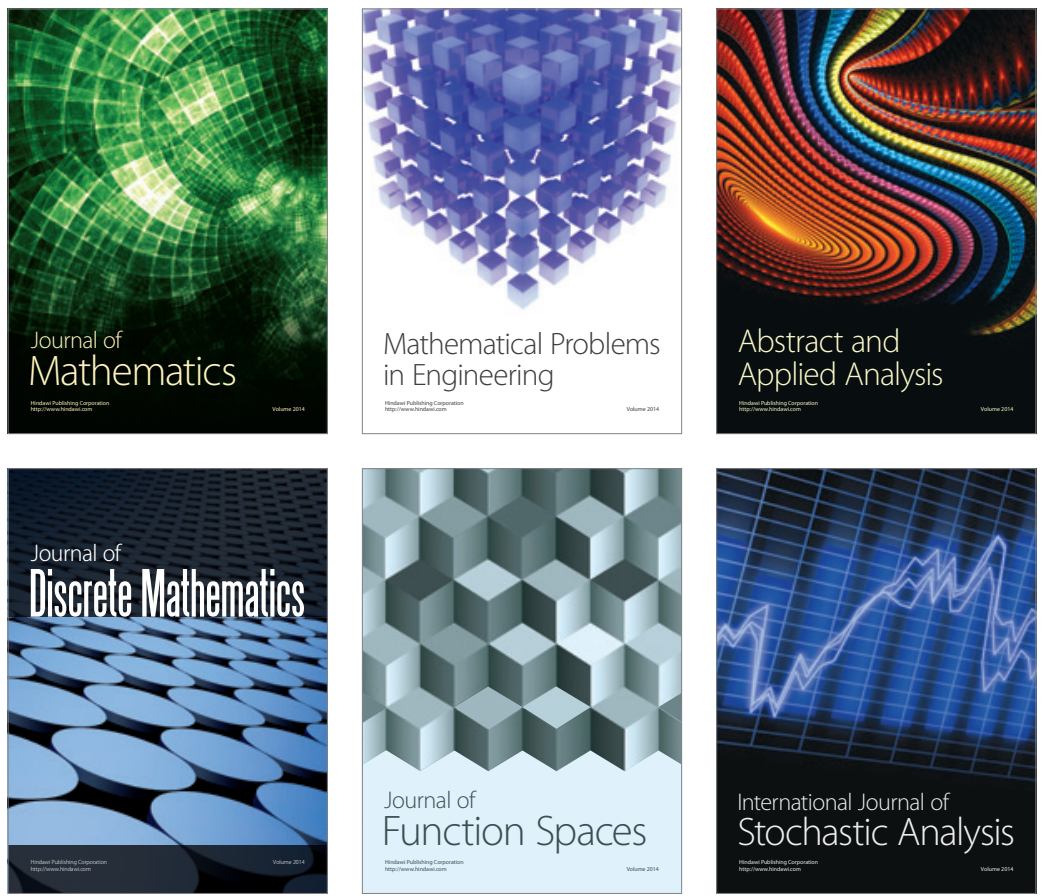

Journal of

Function Spaces

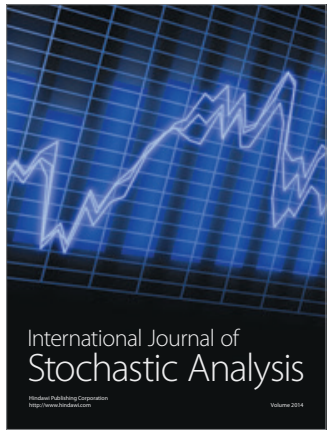

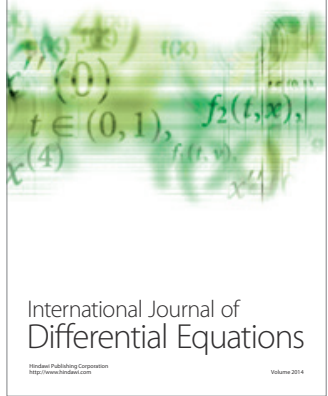
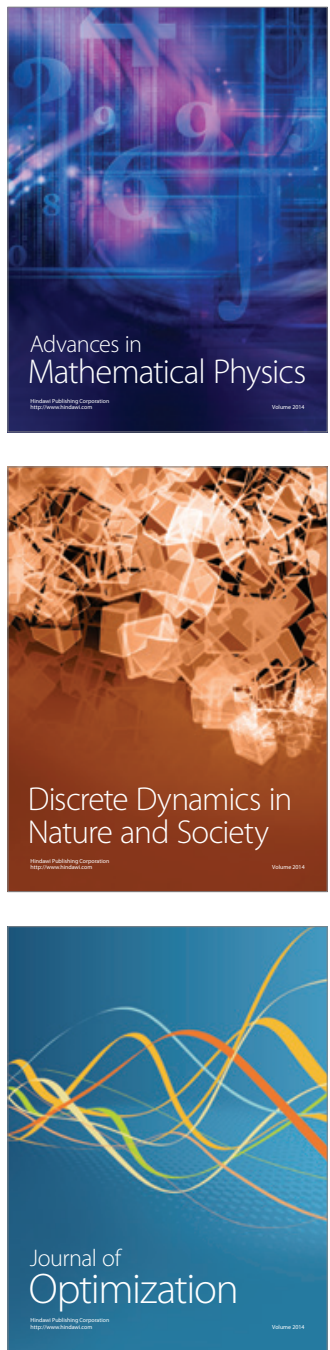